\title{
Adelige Herrschaftsrepräsentation und Memoria im Mittelalter
}

\author{
Einführung $^{1}$
}

Von Peter Rückert

Mit „Adeliger Herrschaftsrepräsentation und Memoria im Mittelalter“ werden zwei aktuelle Leuchtbegriffe mediaevistischer Forschung verbunden. Ihre konkrete Veranschaulichung in der historischen Landschaft an Main und Tauber, in ihren kulturellen Zeugnissen, in Kunst und Architektur, soll im Folgenden den Ansatz für den wissenschaftlichen Diskurs bieten. Zentral im Blickfeld steht dabei das Zisterzienserkloster Bronnbach - ein ursprünglicher Ort der Memoria, dessen hochmittelalterlichen Anfänge auch in seiner heutigen Baugestalt noch beeindruckend greifbar sind (Abb.1). Daneben sind Burg und Stadt Wertheim als monumentale Schauplätze adeliger Geschichte und Herrschaftsrepräsentation bekannt ${ }^{2}$. Dazu tritt die Gamburg, ebenfalls nur wenige Kilometer entfernt gelegen, die mit ihrer bemerkenswerten Erhaltung und aufsehenerregenden Ausstattung besondere bau- und kunstgeschichtliche Bedeutung genieß $\mathrm{t}^{3}$.

Der Sektion der folgenden Beiträge liegt also eine Gesamtkonzeption zugrunde, welche diese beiden wesentlichen Züge mittelalterlicher Geschichte - die „weltliche“ Repräsentation und die „geistliche“ Memoria - neben- und miteinander fokussieren will. Vor allem interessieren dabei deren Berührungen und Überschneidungen. Zunächst geht es darum, sich dem ambitionierten Thema in größeren,

${ }^{1}$ Der Beitrag basiert auf meiner Einführung in die Sektion „Adelige Herrschaftsrepräsentation und Memoria im Mittelalter", die im Rahmen der Jahrestagung der Kommission für geschichtliche Landeskunde in Baden-Württemberg am 28.6.2013 in Wertheim-Bronnbach gestaltet wurde. Der Vortragsstil wurde beibehalten, der Text um den wissenschaftlichen Apparat ergänzt.

2 Vgl. zuletzt dazu Volker Rödes, Immer auch ein Teil des Ganzen? Die wertheimische im Verband der nationalen Geschichte, in: Wertheimer Jahrbuch 2012 (2013) S. 53-84. Diesem Aufsatz lag der Abendvortrag der genannten Kommissionstagung zugrunde.

3 Als Abschluss der Kommissionstagung bot Baron Goswin von Mallinckrodt eine Führung auf der Gamburg an, die eine intensive Diskussion um die neu entdeckten Wandmalereien und Bauplastik anregte und deren weitere kunst- und baugeschichtliche Erforschung als Desiderat erscheinen lässt. Der Familie von Mallinckrodt sei herzlich für die freundliche Aufnahme in ihrer Burg gedankt. 
allgemeineren Kontexten zu nähern, um dann auch mit den örtlichen Konfigurationen konkrete Anhaltspunkte zu finden - daher vorweg einige allgemeiner gehaltene Vorbemerkungen zum Forschungsstand und zu aktuellen Fragen, zur methodischen Herangehensweise und damit der konzeptionellen Ausrichtung der nachfolgenden Beiträge.

Zunächst soll der interdisziplinäre Zugang zum Thema kurz skizziert werden: Ambitionen adeliger Herrschaftsrepräsentation äußern sich im Mittelalter bekanntlich durch monumentale Architektur und ihre Gestaltung bzw. Ausstattung wie durch künstlerische und literarische Selbstdarstellung und repräsentative Inszenierung höfischer Rituale. In den letzten Jahrzehnten haben auch die Literaturgeschichte wie die Kunst- und Baugeschichte die höfische Kultur gerade des Hochmittelalters im Hinblick auf ihre sozialgeschichtlichen Bedingungen und Strukturen, auf ihre repräsentativen und kommunikativen Funktionen beleuchtet. Aktuelle Fragestellungen der gemeinsamen kulturhistorischen Forschung widmen sich verstärkt den mit expliziten Schriftzeugnissen nur dünn besetzten Problemfeldern um Rituale, Repräsentation und Gruppenbildung - moderner: Networking - in der adeligen Gesellschaft ${ }^{4}$.

Damit fällt auch die Gestaltung der Memoria in den Blick, die nicht nur Hinweise auf ihre Träger, auf die sozialen Bindungen von „Verwandten, Freunden und Getreuen" - wie dies Jürgen Dendorfer genannt hat ${ }^{5}$ - bieten kann, sondern natürlich auch repräsentativen Charakter hat ${ }^{6}$. Die Memoria, das Gedenken bzw. rituelle „Erinnern“ ist mit den Forschungen von Otto Gerhard Oexle ${ }^{7}$ zu einem Zentral-

${ }^{4}$ Hierzu sei nur beispielhaft auf einige zentrale Publikationen verwiesen: zur Ritualforschung vor allem Gerd Althoff, Die Macht der Rituale. Symbolik und Herrschaft im Mittelalter, Darmstadt 2003; daneben auch die Sammelbände: Spektakel der Macht. Rituale im alten Europa 800-1800, hg. von Barbara Stollberg-Rillinger/Matthias Puhle/Jutta Götzmann/Gerd Althoff, Darmstadt 2008; Die Welt der Rituale. Von der Antike bis heute, hg. von Claus Амвos/Stephan Hotz/Gerald Schwedler/Stefan Weinfurter, Darmstadt 2006; Inszenierung und Ritual in Mittelalter und Renaissance, hg. von Andrea von Hülsen-Esch (Studia humaniora, Bd. 40), Düsseldorf 2005. Dazu aus literaturwissenschaftlicher Sicht: Joachim Bumke, Höfische Kultur, Literatur und Gesellschaft im hohen Mittelalter, München 2005; aus sozial- und kunsthistorischer Sicht: Die Repräsentation der Gruppen: Texte-Bilder-Objekte, hg. von Otto Gerhard OexLE/Andrea von Hülsen-Esch (Veröffentlichungen des Max-Planck-Instituts für Geschichte, Bd.141), Göttingen 1998.

${ }^{5}$ Jürgen Dendorfer, Verwandte, Freunde und Getreue - Adelige Gruppen in der klösterlichen Memoria des 12. Jahrhunderts in Bayern, in: Adlige - Stifter - Mönche. Zum Verhältnis zwischen Klöstern und mittelalterlichem Adel (Veröffentlichungen des Max-PlanckInstituts für Geschichte, Bd.227), Göttingen 2007, S. 63-105.

${ }^{6}$ Siehe hierzu auch Karl-Heinz SPIEss, Liturgische Memoria und Herrschaftsrepräsentation im nichtfürstlichen Adel des Spätmittelalters, in: Adelige und bürgerliche Erinnerungskulturen des Spätmittelalters und der Frühen Neuzeit, hg. von Werner Rösener (Formen der Erinnerung, Bd. 8), Göttingen 2000, S. 97-123.

7 Memoria als Kultur, hg. von Otto Gerhard Oexle (Veröffentlichungen des Max-PlanckInstituts für Geschichte, Bd.121), Göttingen 1995; Ders., Memoria, Memorialüberlieferung, in: LexMA 6 (1993) Sp. 510-513. 
begriff der internationalen Mediaevistik avanciert und lässt verschiedene Formen und Typen bis zur sogenannten „sozialen Memoria“ unterscheiden, dem gemeinsamen bzw. gegenseitigen Gedenken und Erinnern miteinander verbundener Menschen und Gruppen. Die mit diesem Gedenken zunächst beauftragten geistlichen Institutionen und Gemeinschaften, vor allem Klöster und Stifte, werden seit langem untersucht, die Verbindungen der mittelalterlichen Adelsgesellschaft mit den Orten und Formen ihrer Memoria haben bekanntlich konstitutiven Charakter, oder um es konkreter ins Bild zu setzen: Burg und Kloster stehen gemeinsam für die dynastische Formierung adeliger Herrschaft mit der dieser Gesellschaft immanenten Symbiose von herrschaftlicher Repräsentation und Memoria. Hierzu brauche ich für den deutschen Südwesten nur auf die grundlegenden Forschungen von Karl Schmid ${ }^{8}$ und Hansmartin Schwarzmaier ${ }^{9}$ aufmerksam zu machen, oder auf die Vorträge der Jahrestagung der Kommission für geschichtliche Landeskunde in Baden-Württemberg zu den Welfen 2005 in Weingarten, wo dieser Forschungsstand exemplarisch und programmatisch bestimmt wurde ${ }^{10}$.

Mittlerweile werden große Memoria-Projekte weit über einzelne geistliche Institutionen und Dynastien hinaus international und im virtuellen Raum betrieben. Die einschlägige Online-Datenbank „Medieval Memoria Online“ (MeMo) mehrerer niederländischer Universitäten wird so gerade mit Bild- und Textquellen intensiv und europaweit ausgebaut ${ }^{11}$.

Ausgehend von der aktuellen Diskussion um adelige Memoria und ihren Forschungsproblemen ergeben sich einige konkrete Fragestellungen: Welche sozialen Bindungen und Strukturen können über die Memorialzeugnisse gerade für den deutschen Südwesten erschlossen werden? Wer wurde mit der Pflege der Memoria von wem beauftragt? Hierbei spielen natürlich herrschaftliche Bindungen wie die Frage nach Gründerfamilien, Wohltätern und Vögten nach wie vor eine zentrale Rolle. Zu hinterfragen ist damit aber auch die Bedeutung der „Hausklöster“ nicht nur für die dynastische Formierung, sondern darüber hinaus für die Gruppenbildung im hochmittelalterlichen Adel. Konnte in diesem Sinne - wenn die dynastische Formierung misslang - Memoria auch scheitern? Jürgen Dendorfer fragt im Folgenden nach „gescheiterter Memoria“ und wird „Hausklöster“ des hochmittelalterlichen Adels im deutschen Südwesten in den Mittelpunkt seiner Ausführungen stellen ${ }^{12}$.

${ }^{8}$ Genannt sei hier nur sein richtungsweisender Aufsatz: Karl Schmid, Zur Problematik von Familie, Sippe und Geschlecht, Haus und Dynastie beim mittelalterlichen Adel. Vorfragen zum Thema „Adel und Herrschaft im Mittelalter“, in: ZGO 105 (1957) S. 1-62.

9 Hansmartin Schwarzmaier, Klöster, Stifter, Dynastien. Studien zur Sozialgeschichte des Adels im Hochmittelalter, hg. von Konrad Krimm/Peter RücKert (VKgL B 190), Stuttgart 2012.

10 Vgl. Bernd Schneidmüller/Matthias Becher/Thomas Zotz/Werner Hechberger, Die Welfen. Adelsentwürfe im hohen Mittelalter, in: ZWLG 66 (2007) S. 11-62.

11 http://memo.hum.uu.nl (letzter Zugriff: 4.2.2014).

12 Vgl. den Beitrag von Jürgen Dendorfer in diesem Band, S. 17-38. 
Anschließend soll der wissenschaftliche Blick auf den historischen Raum an Main und Tauber fixiert werden. Als maßgebliche Herrschaftsdynastie prägten die Grafen von Wertheim dessen Geschichte vom 12. bis ins 19. Jahrhundert ${ }^{13}$. Sie bildeten hier gleichsam den Mikrokosmos eines prototypischen dynastischen Profils. Die Orte ihrer Herrschaftsrepräsentation, zunächst Burg und Stadt Wertheim, bestechen heute noch durch ihre architektonische Qualität und können konkrete Eindrücke ihrer mittelalterlichen Gestalt vermitteln. Die Wertheimer Burg gilt mittlerweile sogar als ein „kulturelles Zentrum“ des Hochmittelalters, wird doch auch der höfischen Literatur ein prominenter Platz hinter ihrer anspruchsvollen romanischen Architektur zugesprochen ${ }^{14}$ (Abb. 2).

Wir erkennen hier Wolfram von Eschenbach als Lehensmann des Grafen Poppo von Wertheim, den er im „Parzival“ als seinen Herrn bezeichnet, und wir können zahlreiche topografische Hinweise in Wolframs Werken auf die nähere mainfränkische Umgebung beziehen ${ }^{15}$. Auch wissen wir von einem adeligen Netzwerk miteinander verwandter und befreundeter Familien um die Grafen von Wertheim, die um 1200 den Grafenhof wie die benachbarte Gamburg zu Schauplätzen gemeinsamer herrschaftlicher Repräsentation machten ${ }^{16}$. Als Auftraggeber und Mäzene von Baumeistern, Malern und Dichtern zeigen diese Adelsgruppen gemeinsame repräsentative Ansprüche, die sich in elitären Formen von Architektur und Literatur greifen lassen, die auf Außenwirkung angelegt sind.

Komplementär dazu gestalteten die Grafen von Wertheim und die mit ihnen verbundenen Adelsherren im Tauberland eine aufwändige Memorialkultur, die auch ihre profanen Lebensbereiche mit einbezog ${ }^{17}$ : Offenbar wesentlich angeregt durch persönliche Erlebnisse während der Kreuzzüge, wurde ihre Erinnerung gemeinsam zelebriert und auch in architektonischen und bildlichen Darstellungen vergegenwärtigt. Die Ausmalung des Palas auf der Gamburg mit einer Darstellung zum

${ }^{13}$ Vgl. dazu den aktuellen Überblick von Volker RödeL, Wertheim, in: Höfe und Residenzen im spätmittelalterlichen Reich. Grafen und Herren, Teilband 2, hg. von Werner PARAvicini, Ostfildern 2012, S. 1649-1662, sowie Hermann Ehmer, Geschichte der Grafschaft Wertheim, Wertheim 1989.

${ }^{14}$ Hierzu zuletzt Peter Rückert, Die Grafen von Wertheim und ihr Hof um 1200: eine einführende Skizze, in: Wertheimer Jahrbuch 2008/2009 (2010) S. 17-26.

${ }^{15} \mathrm{Vgl}$. Horst Brunner, Wolfram von Eschenbach und der Graf von Wertheim, in: ebd., S. 27-40.

16 Ausführlicher dazu Peter Rückert, Adelige Herrschaft und Repräsentation im hohen Mittelalter. Literatur und Architektur im Umfeld der Grafen von Wertheim und der Herren von Gamburg, in: Wirtschaft - Gesellschaft - Mentalitäten im Mittelalter. Festschrift zum 75. Geburtstag von Rolf Sprandel, hg. von Hans-Peter BAum/Rainer Leng/Joachim SchneIDER (Beiträge zur Sozial- und Wirtschaftsgeschichte, Bd. 107), Stuttgart 2006, S. 289-306.

$17 \mathrm{Zu}$ den miteinander verwandten Adelsherren im Tauberland vgl. Peter Rückert, Die Edelfreien von Gamburg, Lauda und Zimmern, in: Hochmittelalterliche Adelsfamilien in Altbayern, Franken und Schwaben, hg. von Ferdinand Kramer/Wilhelm Störmer, München 2005, S. 591-642. 
3. Kreuzzug erkennen wir vor diesem Hintergrund als außergewöhnliches Zeugnis adeliger Erinnerungskultur.

Über die geistliche Pflege der Memoria für die Grafen von Wertheim sind wir in dieser Frühzeit allerdings kaum unterrichtet. Frühe Kontakte zu der nahen Fuldaer Propstei Holzkirchen werden im späten 12. Jahrhundert offenbar abgelöst durch Wohltaten für die junge Zisterze Bronnbach ${ }^{18}$. Der Beitrag von Hermann Ehmer wird die Grafen von Wertheim und ihre Memoria über das Mittelalter hinweg genauer vorstellen ${ }^{19}$. Damit steht zunächst wieder Bronnbach im Blickpunkt, als ihre zeitweilige Grablege und geistiges Zentrum für die Familie. Wir werden das Zisterzienserkloster auch darüber hinaus als programmatischen Memorialort ansprechen können: Zunächst für die Klosterstifter, eine Reihe miteinander verwandter Edelfreier um die Herren von Gamburg - wovon allerdings laut klösterlicher Überlieferung keiner in Bronnbach begraben sein soll. Und dann für die wohl prominenteste Gestalt der Bronnbacher Frühgeschichte: Erzbischof Arnold von Mainz, der zu den großen Wohltätern Bronnbachs im Umfeld seiner Gründung gehörte und sich - laut seiner Vita - das Kloster als Begräbnisort und zur Pflege seiner Memoria ausgesucht hatte.

Auch dazu sollte es nicht kommen, und doch wird die ungewöhnliche und anspruchsvolle Gestalt der Klosterkirche, die damals im Bau war, aus Sicht der Architekturgeschichte gerade als monumentales Memorialzeugnis für den Mainzer Erzbischof Arnold gelesen ${ }^{20}$. Damit wäre geistliche Herrschaft und Memoria in Bronnbach eine repräsentative Bindung eingegangen, und der ambitionierte Erzbischof Arnold kann - auch ohne leibliche Präsenz - den aufgezeigten Problemkreis nicht nur abrunden, sondern zu neuen Fragestellungen hin öffnen. Diese wird der Beitrag von Stefan Weinfurter aufnehmen, der gerade auch den Verfasser von Arnolds Vita vorstellen wird, die zunächst nach dessen Memoria zu befragen ist ${ }^{21}$. Und steht schließlich nicht auch diese tendenziöse Darstellung für eine Verbindung von Herrschaftsrepräsentation und Memoria? - Somit nehmen die folgenden Beiträge und ihre Diskussion nicht zuletzt Teil an der örtlichen Tradition um das Kloster Bronnbach und die Grafen von Wertheim und führen diese im wissenschaftlichen Diskurs weiter hinaus, angeregt und vergewissert durch die presentia loci.

18 Vgl. zu Bronnbach Leonhard Scherg, Die Zisterzienserabtei Bronnbach im Mittelalter. Studien zur Geschichte der Abtei von der Gründung bis zur Mitte des 14. Jahrhunderts (Mainfränkische Studien 14), Würzburg 1976.

19 Vgl. den Beitrag von Hermann Ehmer in diesem Band, S. 39-58.

20 Dietlinde Schmitt-Vollmer, Bronnbach. Ein Grablegeprojekt im 12. Jahrhundert. Zur Baugeschichte der Zisterzienserkirche (Forschungen und Berichte der Bau- und Kunstdenkmalpflege in Baden-Württemberg, Bd.12), 2 Bde., Stuttgart 2007. Und zuletzt dazu: Katinka KRUG, Kloster Bronnbach. Die Baugeschichte von Kirche und Klausur des Zisterzienserklosters (Forschungen und Berichte der Bau- und Kunstdenkmalpflege in Baden-Württemberg, Bd. 15), Stuttgart 2012.

21 Vgl. den Beitrag von Stefan Weinfurter in diesem Band, S. 59-71. 
Abb. 1: Luftbild des Klosters Bronnbach

(Vorlage: Eigenbetrieb Kloster Bronnbach, 2009).

Abb. 2: Die Ruine der Burg Wertheim mit dem stauferzeitlichen Palas neben dem Bergfried (Aufnahme: Schrenk-Verlag, Gunzenhausen).

[Die Abbildungen, im Buch auf einer Farbtafel nach S. 16, können aus rechtlichen Gründen online nicht bereitgestellt werden.] 